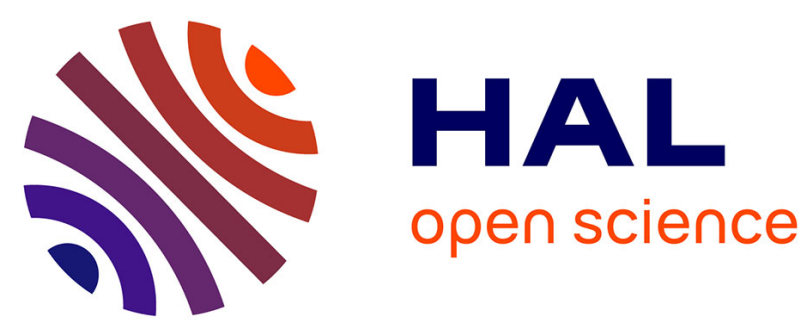

\title{
Minimizing the second eigenvalue of the Laplace operator with Dirichlet boundary conditions
}

Antoine Henrot, Edouard Oudet

\section{To cite this version:}

Antoine Henrot, Edouard Oudet. Minimizing the second eigenvalue of the Laplace operator with Dirichlet boundary conditions. Archive for Rational Mechanics and Analysis, 2003, 169, pp.73-87. 10.1007/s00205-003-0259-4 . hal-00115319

\section{HAL Id: hal-00115319 https://hal.science/hal-00115319}

Submitted on 21 Nov 2006

HAL is a multi-disciplinary open access archive for the deposit and dissemination of scientific research documents, whether they are published or not. The documents may come from teaching and research institutions in France or abroad, or from public or private research centers.
L'archive ouverte pluridisciplinaire HAL, est destinée au dépôt et à la diffusion de documents scientifiques de niveau recherche, publiés ou non, émanant des établissements d'enseignement et de recherche français ou étrangers, des laboratoires publics ou privés. 


\title{
ARMA manuscript No.
}

(will be inserted by the editor)

\section{Minimizing the second eigenvalue of the Laplace operator with Dirichlet boundary conditions}

\author{
Antoine HENROT, Edouard OUdET
}

\begin{abstract}
In this paper, we are interested in the minimization of the second eigenvalue of the Laplacian with Dirichlet boundary conditions amongst convex plane domains with given area. The natural candidate to be the optimum was the "stadium", convex hull of two identical tangent disks. We refute this conjecture. Nevertheless, we prove existence of a minimzer. We also study some qualitative properties of the minimizer (regularity, geometric properties).
\end{abstract}

\section{Introduction}

Let $\Omega$ be a bounded open subset in the plane and let us denote by $0<\lambda_{1}(\Omega) \leq \lambda_{2}(\Omega) \leq \lambda_{3}(\Omega) \ldots$ its eigenvalue for the Laplacian operator with homogeneous Dirichlet boundary condition. Problems of minimization of eigenvalues, or combination of eigenvalues, brought about many deep works since the early part of the twentieth century. It was indeed in the 1920's that Faber [12] and Krahn [21] solved the well-known Rayleigh's conjecture: the ball minimizes $\lambda_{1}$ among every open sets of given measure. The same question for $\lambda_{2}$ was solved (to our knowledge) by Szegö, cf [27] (it seems nevertheless that the result was already contained in one of the paper of Krahn [22]): the open set which minimizes $\lambda_{2}$ is the union of two identical balls. For sake of completeness, the proof is given in section 2. Looking for the minimizer of $\lambda_{2}$ among connected domains has no solution. Actually, it is easy to see (cf section 2) that the domains obtained by joining the two balls with a thin pipe provide a minimizing sequence whose second eigenvalue converges to the second eigenvalue of the two balls, so the infimum is not achieved.

Now, the problem becomes again interesting if we ask the question to find the convex domain, of given area, which minimizes $\lambda_{2}$. Existence of a 
minimizer $\Omega^{*}$ is easy to obtain (see [9] and Theorem 4). In a paper of 1973 [31], Troesch did some numerical experiments which led him to conjecture that the solution was a stadium: convex hull of two identical tangent disks. It is actually the convex domain which is the closer to the solution without convexity constraint. In this paper, we refute this conjecture:

Theorem 1. The stadium, convex hull of two identical tangent disks, does not realize the minimum of $\lambda_{2}$ among plane convex domains of given area.

Let us remark that the method of the proof also gives an answer to a question set by Giuseppe Buttazzo: if we minimize $\lambda_{1}(\Omega)$ among open sets $\Omega$ of given area $c$, contained in a fixed box $D$ and if $D$ is too small to contain a ball of area $c$, are the free parts of the boundary of $\Omega$ pieces of spheres? The answer is NO, using the same argument, see proof of Theorem 8.

Coming back to the second eigenvalue, the minimizer looks like very much a stadium! In section 3, we give the following properties of the minimizer:

Theorem 2. The following facts hold:

Regularity The minimizer $\Omega^{*}$ is at least $C^{1}$ and at most $C^{2}$.

Simplicity If we assume $\Omega^{*}$ of class $C^{1,1}$ then the second eigenvalue of $\Omega^{*}$ is simple.

Geometry If we assume $\Omega^{*}$ of class $C^{1,1}$ then it has two (and only two) straight lines in its boundary and these lines are parallels.

Some of these results were already announced in the Note [18]. To be complete, it would be nice to prove also that $\Omega^{*}$ has two orthogonal axis of symmetry. Numerical experiments, see [25] seem to show that it is the case, but we failed in proving it. For other recent results on minimization problems for functions of eigenvalues, we refer e.g. to [5], [32] and the review papers [2], [4], [17]. Finally, we point out that the results in section 2 are mostly valid in any dimension while the results of the section 3 are more specifically two-dimensional.

\section{General results}

We first recall some classical results involving eigenvalues of the LaplaceDirichlet operator, we refer to classical books like Courant-Hilbert, [8] or Dautray-Lions, vol.5, [11].

Let $\Omega$ be a bounded open subset in $\mathbb{R}^{N}$, we denote by $0<\lambda_{1}(\Omega) \leq$ $\lambda_{2}(\Omega) \leq \lambda_{3}(\Omega) \ldots$ its eigenvalue for the Laplacian operator with homogeneous Dirichlet boundary condition and $u_{1}, u_{2}, u_{3}, \ldots$ the corresponding eigenfunction. They solve

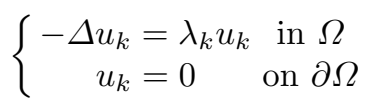


The sequence $\left(u_{k}\right)_{k \geq 1}$ is a Hilbert basis of the space $L^{2}(\Omega)$. Each eigenfunction $u_{k}$ is analytic inside $\Omega$, its behavior on the boundary is governed by classical regularity results for elliptic partial differential equations. For example, if the boundary of $\Omega$ is of class $C^{1,1}$, it can be proved that $u_{k}$ is $C^{1}$ up to the boundary, see [13] or [14]. For a functional point of view, the functions $u_{k}$ belong to the Sobolev space $H_{0}^{1}(\Omega)$ defined as the closure of the space $\mathcal{D}(\Omega)$ of infinitely differentiable functions with compact support for the norm

$$
\|u\|_{H^{1}}:=\left(\int_{\Omega} u^{2}(x) d x+\int_{\Omega}|\nabla u(x)|^{2} d x\right)^{1 / 2} .
$$

The first eigenfunction $u_{1}$ is non-negative in $\Omega$. Moreover, if $\Omega$ is connected, $u_{1}$ is positive and $\lambda_{1}(\Omega)$ is simple (this is a consequence of the Krein-Rutman Theorem). Since $u_{2}$ is orthogonal to $u_{1}$, it has to change of sign in $\Omega$. The sets

$$
\Omega_{+}=\left\{x \in \Omega, u_{2}(x)>0\right\} \quad \text { and } \quad \Omega_{-}=\left\{x \in \Omega, u_{2}(x)<0\right\}
$$

are called the nodal domains of $u_{2}$. According to the Courant-Hilbert Theorem, these two nodal domains are connected subsets of $\Omega$. The set

$$
\mathcal{N}=\left\{x \in \Omega, u_{2}(x)=0\right\}
$$

is called the nodal line of $u_{2}$. When $\Omega$ is a plane convex domain, this nodal line hits the boundary of $\Omega$ at exactly two points, see Melas [24], or Alessandrini [1]. For general simply connected plane domains $\Omega$, it is still a conjecture, named after Larry Payne, the "Payne conjecture". The classical min-max formula of Courant-Fischer for eigenvalues implies the following monotonicity for inclusion:

$$
\Omega_{1} \subset \Omega_{2} \Longrightarrow \lambda_{k}\left(\Omega_{1}\right) \geq \lambda_{k}\left(\Omega_{2}\right)
$$

At last, the eigenvalues have a simple behavior with respect to homothety: if $t \Omega$ denotes the image of $\Omega$ by an homothety with ratio $t$, the eigenvalues of $t \Omega$ satisfy:

$$
\lambda_{k}(t \Omega)=\frac{\lambda_{k}(\Omega)}{t^{2}} .
$$

As a consequence, in two-dimensions, looking for the minimizer of $\lambda_{k}(\Omega)$ with a volume constraint is equivalent to look for a minimizer of the product $|\Omega| \lambda_{k}(\Omega)$. In the sequel, we will use one or the other of these formulations. At last, we will generically denote by $n$ the exterior unit normal vector to the boundary of $\Omega$.

We first recall the result about the minimization of the second eigenvalue:

Theorem 3 (Krahn-Szegö). The minimum of $\lambda_{2}(\Omega)$ among bounded open sets of $\mathbb{R}^{N}$ with given volume is achieved by the union of two identical balls. 
Proof : Let $\Omega$ be any bounded open set, and let us denote by $\Omega_{+}$and $\Omega_{-}$ its nodal domains. Since $u_{2}$ satisfies

$$
\left\{\begin{array}{cc}
-\Delta u_{2}=\lambda_{2} u_{2} & \text { in } \Omega_{+} \\
u_{2}=0 & \text { on } \partial \Omega_{+}
\end{array}\right.
$$

$\lambda_{2}(\Omega)$ is an eigenvalue for $\Omega_{+}$. But, since $u_{2}$ is positive in $\Omega_{+}$, it is the first eigenvalue (and similarly for $\Omega_{-}$):

$$
\lambda_{1}\left(\Omega_{+}\right)=\lambda_{1}\left(\Omega_{-}\right)=\lambda_{2}(\Omega) .
$$

We now introduce $\Omega_{+}^{*}$ and $\Omega_{-}^{*}$ the balls of same volume as $\Omega_{+}$and $\Omega_{-}$ respectively. According to the Rayleigh-Faber-Krahn inequality

$$
\lambda_{1}\left(\Omega_{+}^{*}\right) \leq \lambda_{1}\left(\Omega_{+}\right), \quad \lambda_{1}\left(\Omega_{-}^{*}\right) \leq \lambda_{1}\left(\Omega_{-}\right) .
$$

Let us introduce a new open set $\tilde{\Omega}$ defined as

$$
\tilde{\Omega}=\Omega_{+}^{*} \cup \Omega_{-}^{*} .
$$

Since $\tilde{\Omega}$ is disconnected, we obtain its eigenvalues by gathering and reordering the eigenvalues of $\Omega_{+}^{*}$ and $\Omega_{-}^{*}$. Therefore,

$$
\lambda_{2}(\tilde{\Omega}) \leq \max \left(\lambda_{1}\left(\Omega_{+}^{*}\right), \lambda_{1}\left(\Omega_{-}^{*}\right)\right) .
$$

According to (2), (3) we have

$$
\lambda_{2}(\tilde{\Omega}) \leq \max \left(\lambda_{1}\left(\Omega_{+}\right), \lambda_{1}\left(\Omega_{-}\right)\right)=\lambda_{2}(\Omega) .
$$

This shows that the minimum of $\lambda_{2}$ is to be obtained among the union of balls. But, if the two balls would have different radii, we would decrease the second eigenvalue by shrinking the largest one and dilating the smaller one (without changing the total volume). Therefore, the minimum is achieved by the union of two identical balls.

As we said in the Introduction, a connectedness constraint does not really change the situation. Indeed, let us consider the following domain (see Figure 1) $\Omega_{\varepsilon}$, obtained by joining the union of the two previous balls $\Omega$ by a thin pipe of width $\varepsilon$. We say that $\Omega_{\varepsilon} \gamma$-converges to $\Omega$ if the resolvant operators $T_{\varepsilon}$ associated to the Laplace-Dirichlet operator on $\Omega_{\varepsilon}$ simply converge to the corresponding operator $T$ on $\Omega$, see e.g. [10]. By compactness argument, see [7], [19] it can be proved that this simple convergence implies the convergence in norm of operators and therefore the convergence of eigenvalues. Now, it is easy to verify, see [6], [19], that in the above situation $\Omega_{\varepsilon} \gamma$-converges to $\Omega$ what yields $\lambda_{2}\left(\Omega_{\varepsilon}\right) \rightarrow \lambda_{2}(\Omega)$ and therefore:

$\inf \left\{\lambda_{2}(\Omega), \Omega \subset \mathbb{R}^{N}, \Omega\right.$ connected,$\left.|\Omega|=c\right\}=\min \left\{\lambda_{2}(\Omega), \Omega \subset \mathbb{R}^{N},|\Omega|=c\right\}$

what shows that this infimum is not achieved (actually, we can prove that the union of two balls is the unique minimizer of $\lambda_{2}$ up to displacements and zero-capacity subsets). 


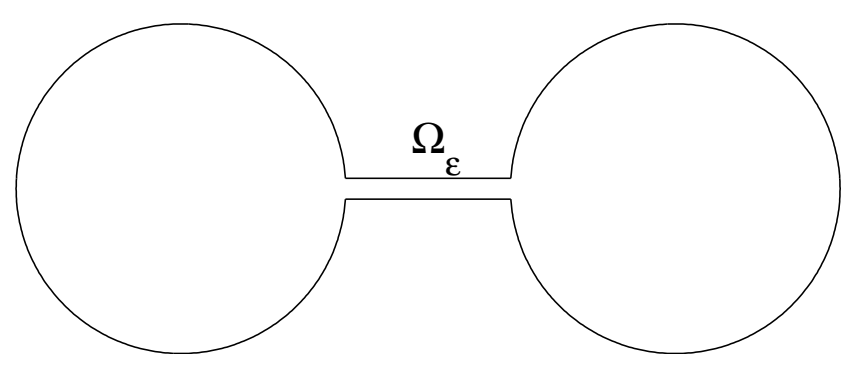

Fig. 1. A minimizing sequence of connected domains

In the sequel of this paper, we are dealing with convex domains. First of all, we give an existence result for the problem of minimization of any eigenvalue among convex domains of given volume. This result is probably already known, but we give here a proof for sake of completeness. The two-dimensional version of this result is already contained in a paper of Cox-Ross, [9], where the proof uses a clever and explicit estimate between the difference of the eigenvalues of two distinct domains with respect to their geometry. Our proof below is less explicit, but more general.

Theorem 4. Let $c$ be a positive constant and $k$ any integer. Then there exists a convex domain $\Omega^{*}$ such that

$$
\lambda_{k}\left(\Omega^{*}\right)=\min \left\{\lambda_{k}(\Omega), \Omega \subset \mathbb{R}^{N}, \Omega \text { convex },|\Omega|=c\right\} .
$$

Proof : We use the classical method of calculus of variations. Let $\Omega_{n}$ be a minimizing sequence. First of all, we claim that the diameters of the $\Omega_{n}$ are bounded. Indeed, let us define the width of $\Omega_{n}$ as the maximum diameter of any section of $\Omega_{n}$ with an hyperplane orthogonal to the segment which realizes the diameter. Then, by convexity property and the fact that all the domains $\Omega_{n}$ have a given volume, if the diameters of $\Omega_{n}$ would not be bounded, the width of $\Omega_{n}$ would have to go to zero. Therefore, we could find a sequence of parallelepipeds (or cylinders), containing $\Omega_{n}$ and with, at least, one dimension going to zero. But for such parallelepipeds, the value of $\lambda_{1}$ is known and it goes to $+\infty$. Therefore, by the monotonicity property, we would have $\lambda_{k}\left(\Omega_{n}\right) \rightarrow+\infty$.

Since the diameters are bounded, we can assume (up to a translation) that the whole sequence is contained in a fixed ball, say $B$. We use now standard arguments of optimal design, see e.g. [19], [26], [6]. By classical property of the Hausdorff convergence, there exists a convex domain $\Omega^{*}$ and a subsequence, still denoted by $\Omega_{n}$ such that $\Omega_{n}$ converges to $\Omega^{*}$ for the Hausdorff metric (of the complementaries in $B$ ). Each domains being convex, it is easy to see that the characteristic functions of $\Omega_{n}$ converge to the characteristic function of $\Omega^{*}$ in $L^{1}(B)$. In particular, $\left|\Omega_{n}\right| \rightarrow\left|\Omega^{*}\right|$ and $\left|\Omega^{*}\right|=c$. At last, we can also prove using stability arguments, see [19], [16] or [20] that the sequence $\Omega_{n} \gamma$-converges to $\Omega^{*}$. This implies, see above, the 
convergence of the eigenvalues and then $\Omega^{*}$ achieves the minimum of $\lambda_{k}$. $\square$

We now give a result that is somewhat related to the genericity of domains with simple eigenvalues. It will imply that for the optimal domain $\Omega^{*}$, the corresponding eigenvalue $\lambda_{2}\left(\Omega^{*}\right)$ is simple. We state the next Lemma in the context of convex domains, but it is true without this geometric restriction.

Lemma 1. Let $\Omega$ be a convex domain of class $C^{1,1}$. We assume that $\Omega$ has a multiple eigenvalue of order $m$ :

$$
\lambda_{k+1}(\Omega)=\lambda_{k+2}(\Omega)=\ldots=\lambda_{k+m}(\Omega) \quad k \geq 1 .
$$

Then, we can always find a deformation field $V \in C^{1,1}\left(\mathbb{R}^{N}, \mathbb{R}^{N}\right)$, preserving the volume and the convexity and such that, if we set

$$
\Omega_{t}=(I d+t V)(\Omega)
$$

we have, for $t>0$ small enough

$$
\lambda_{k+1}\left(\Omega_{t}\right)<\lambda_{k+1}(\Omega)=\lambda_{k+m}(\Omega)<\lambda_{k+m}\left(\Omega_{t}\right) .
$$

Remark 1. The previous result has the following consequence about minimization of eigenvalues: if $\Omega^{*}$ is a domain minimizing the $k$-th eigenvalue (with or without convexity constraint) and if $\lambda_{k}\left(\Omega^{*}\right)$ is not simple, necessarily we have

$$
\lambda_{k-1}\left(\Omega^{*}\right)=\lambda_{k}\left(\Omega^{*}\right) .
$$

Actually, numerical experiments show that this relation holds in every case, see [25]: the domain which minimizes $\lambda_{k}(\Omega), k \geq 2$ (with a volume constraint but without convexity constraint) always satisfies (4). Coming back to a convex domain $\Omega^{*}$ minimizing $\lambda_{2}$, we know that $\lambda_{1}\left(\Omega^{*}\right)$ is simple and therefore (4) cannot hold. Consequently:

Theorem 5. Let $\Omega^{*}$ be a convex domain minimizing the second eigenvalue $\lambda_{2}$ (among convex domains of given volume). Assume that $\Omega^{*}$ is of class $C^{1,1}$. Then $\lambda_{2}\left(\Omega^{*}\right)$ is simple.

Proof of the Lemma : We use the classical tool of derivative with respect to the domain (or Hadamard formulae), see e.g. [30], [29], [19]. Let us deform the domain $\Omega$ thanks to a deformation field $V$ as described in the statement of the Lemma. In the case of a multiple eigenvalue, this eigenvalue is no longer Frechet differentiable, but nevertheless it admits directional derivatives, i.e. the differential quotients

$$
\frac{\lambda_{k+p}\left(\Omega_{t}\right)-\lambda_{k+p}(\Omega)}{t}, \text { for } p=1, \ldots, m
$$


have a limit when $t$ goes to 0 . Moreover, these limits are the eigenvalues of the $m \times m$ matrix

$$
\mathcal{M}=\left(-\int_{\partial \Omega} \frac{\partial u_{i}}{\partial n} \frac{\partial u_{j}}{\partial n} V \cdot n d \sigma\right)_{k+1 \leq i, j \leq k+m}
$$

where $\frac{\partial u_{i}}{\partial n}$ denotes the normal derivative of the $i$-th eigenfunction $u_{i}$ and $V . n$ is the normal displacement of the boundary induced by the deformation field $V$. For a proof of the above-mentioned result, we refer to [15] or [28].

Let us now choose two points $A$ and $B$ located on strictly convex parts of $\partial \Omega$. Let us consider a deformation field $V$ such that $V . n=1$ in a small neighborhood of $A$ (on the boundary of $\Omega^{*}$ ) of size $\varepsilon, V . n=-1$ in a small neighborhood of $B$ (with same measure) and $V$ regularized outside in a neighborhood of size $2 \varepsilon$ in such a way that $\left|\Omega_{t}\right|=|\Omega|$ (it is always possible since the derivative of the volume is given by $d \mathrm{Vol}=\int_{\partial \Omega} V . n d \sigma$ which vanishes with an appropriate choice of the regularization). Moreover, for $t$ small enough, $\Omega_{t}$ will remain convex since we have deformed $\Omega$ only on a strictly convex part of its boundary (more precisely, we can take the convexification of $\Omega_{t}$ without changing first order terms in the derivative, see below the proof of Theorem 7 for details). According to the above-mentioned results about the directional derivatives, the Lemma will be proved if we can find two points $A, B$ such that the symmetric matrix $\mathcal{M}$ has both positive and negative eigenvalues. Now, when $\varepsilon$ goes to 0 , it is clear that the matrix $\mathcal{M}$ behaves like the $m \times m$ matrix

$$
\mathcal{M}_{A, B}=\left(-\frac{\partial u_{i}}{\partial n}(A) \frac{\partial u_{j}}{\partial n}(A)+\frac{\partial u_{i}}{\partial n}(B) \frac{\partial u_{j}}{\partial n}(B)\right)_{k+1 \leq i, j \leq k+m} .
$$

Let us denote by $\phi_{A}$ (resp. $\left.\phi_{B}\right)$ the vector of components $\frac{\partial u_{i}}{\partial n}(A)$, (resp. $\left.\frac{\partial u_{i}}{\partial n}(B)\right), i=k+1, \ldots, k+m$. A straightforward computation gives, for any vector $X \in \mathbb{R}^{m}$ :

$$
X^{T} \mathcal{M}_{A, B} X=\left(X . \phi_{B}\right)^{2}-\left(X . \phi_{A}\right)^{2} .
$$

Therefore, the signature of the quadratic form defined by $\mathcal{M}_{A, B}$ is $(1,1)$ as soon as the vectors $\phi_{A}$ and $\phi_{B}$ are non colinears. Now, assuming these two vectors to be colinear for every choice of points $A, B$ would give the existence of a constant $c$ such that, on a strictly convex part $\gamma$ of $\partial \Omega$ :

$$
\frac{\partial u_{k+1}}{\partial n}=c \frac{\partial u_{k+2}}{\partial n} .
$$

But, $u_{k+1}-c u_{k+2}$ would satisfy

$$
\left\{\begin{array}{cc}
-\Delta\left(u_{k+1}-c u_{k+2}\right)=\lambda_{k+1}\left(u_{k+1}-c u_{k+2}\right) & \text { in } \Omega \\
u_{k+1}-c u_{k+2}=0 & \text { on } \partial \Omega \cap \gamma \\
\frac{\partial\left(u_{k+1}-c u_{k+2}\right)}{\partial n}=0 & \text { on } \partial \Omega \cap \gamma .
\end{array}\right.
$$

Now, by Hölmgren uniqueness theorem, the previous p.d.e. system is solvable only by $u_{k+1}-c u_{k+2}=0$ (first in a neighborhood of $\gamma$ and then in the whole domain by analyticity) which gives the desired contradiction. 


\section{Description of the convex minimizer}

In this section, we will denote by $\Omega^{*}$ a plane convex domain which minimizes $\lambda_{2}$ the second Dirichlet eigenvalue of the Laplacian among convex domains of given area $c$. As explained at the beginning of the previous section, it is also (up to an homothety) a minimizer of the product $|\Omega| \lambda_{2}(\Omega)$. We will denote by $u_{2}$ (or $u$ when no misunderstanding can occur) the corresponding eigenfunction normalized by $\int_{\Omega^{*}} u^{2}(x) d x=1$.

\subsection{A first regularity result}

We begin by proving that the minimizer is $C^{1}$ : it cannot have a corner. We give the proof for the second eigenvalue, but it is clear that this proof can be extended to the problem of minimizing other eigenvalues with a convex constraint. Indeed, it is a particular case of a general result as it will be shown in [3]. In this paper, D. Bucur proves $C^{1}$ regularity for the boundary of an optimal domain $\Omega^{*}$ of a functional $J$, provided that this functional be Lipschitz continuous for a distance related to the $\gamma$-convergence. It is not obvious (but true) to verify that $\lambda_{2}$ satisfies this property. Our proof below is more simple, but less general. Let us also mention that L. Caffarelli, P.L. Lions on the one hand and G. Carlier, T. Lachand-Robert on the other hand have obtained more precise regularity results $\left(C^{1, \alpha}\right.$ or $C^{1,1}$ regularity) for some other problems of minimization with convexity constraints (works to appear).

Theorem 6. The minimizer $\Omega^{*}$ is (at least) $C^{1}$.

Proof : Let us assume, for a contradiction, that $\Omega^{*}$ has two distinct supporting lines at some point $x_{0}$ of its boundary. Without loss of generality, we can assume that $x_{0}$ is the origin. We are going to prove that we can decrease the product $|\Omega| \lambda_{2}(\Omega)$ by cutting a small cap of size $\varepsilon$. Let us introduce the following notations see Figure 2. We denote by $\alpha<\pi$ the opening angle of the two supporting lines, $\eta$ is the (normalized) inward bissector, $C_{\varepsilon}$ the cap defined as $C_{\varepsilon}=\left\{x \in \Omega^{*}, x . \eta \leq \varepsilon\right\}, \Omega_{\varepsilon}$ is the (convex) domain that we obtain in removing the cap $C_{\varepsilon}: \Omega_{\varepsilon}=\Omega^{*} \backslash C_{\varepsilon}$. We will also need $B_{\varepsilon}=\left\{x \in \Omega^{*}, \varepsilon<x . \eta \leq 2 \varepsilon\right\}, C_{2 \varepsilon}=C_{\varepsilon} \cup B_{\varepsilon}$ and $\Omega_{2 \varepsilon}=\Omega_{\varepsilon} \backslash B_{\varepsilon}=\Omega^{*} \backslash C_{2 \varepsilon}$. We use a variational method to estimate $\lambda_{2}\left(\Omega_{\varepsilon}\right)$. We denote by $u_{1}$ and $u_{2}$ the two first normalized eigenfunctions of $\Omega^{*}$. The key point is the following: by classical barrier arguments (comparison with the eigenvalue of a circular sector), it is well known that $u_{1}$ and $u_{2}$ have a gradient which vanishes at the corner:

$$
\lim _{x \rightarrow 0, x \in \Omega^{*}}\left|\nabla u_{i}(x)\right|=0, \text { for } i=1,2 .
$$

Let $\beta>0$ be a small number (which will be chosen at the end), according to (7) and the mean value theorem, we can choose $\varepsilon$ small enough such that

$$
\forall x \in C_{2 \varepsilon} \quad\left|u_{i}(x)\right| \leq \beta|x|, \text { for } i=1,2 .
$$




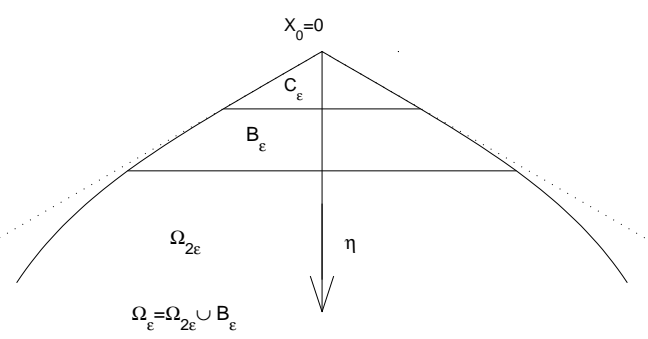

Fig. 2. Removing a cap

In particular, for $i=1,2$ :

$$
\int_{C_{2 \varepsilon}}\left|u_{i}(x)\right|^{2} d x \leq \beta^{2} \int_{C_{2 \varepsilon}}|x|^{2} d x \leq \frac{4 \alpha \beta^{2} \varepsilon^{4}}{\cos ^{4} \alpha / 2} .
$$

We now introduce a $C^{1}$ cut-off function $\chi_{\varepsilon}$ with

$$
\left\{\begin{array}{cc}
\chi_{\varepsilon}(x)=1 & \text { if } x \in \Omega_{2 \varepsilon} \\
0 \leq \chi_{\varepsilon}(x) \leq 1 & \text { if } x \in B_{\varepsilon} \\
\chi_{\varepsilon}(x)=0 & \text { if } x \in C_{\varepsilon}
\end{array}\right.
$$

and two functions belonging to the Sobolev space $H_{0}^{1}\left(\Omega_{\varepsilon}\right)$ :

$$
u_{\varepsilon}^{1}=\chi_{\varepsilon} u_{1} \quad u_{\varepsilon}^{2}=\chi_{\varepsilon} u_{2} .
$$

Since $u_{\varepsilon}^{1}$ and $u_{\varepsilon}^{2}$ are linearly independent, we can consider the two-dimensional subspace $V$ of $H_{0}^{1}\left(\Omega_{\varepsilon}\right)$ spanned by these two functions. According to the Min-max Courant-Fischer formula, we have

$$
\lambda_{2}\left(\Omega_{\varepsilon}\right) \leq \max _{v \in V} \frac{\int_{\Omega_{\varepsilon}}|\nabla v|^{2} d x}{\int_{\Omega_{\varepsilon}} v^{2} d x}=\max _{\left(a_{1}, a_{2}\right) \in \mathbb{R}^{2}} \frac{\int_{\Omega_{\varepsilon}}\left|\nabla\left(a_{1} u_{\varepsilon}^{1}+a_{2} u_{\varepsilon}^{2}\right)\right|^{2} d x}{\int_{\Omega_{\varepsilon}}\left(a_{1} u_{\varepsilon}^{1}+a_{2} u_{\varepsilon}^{2}\right)^{2} d x}
$$

Now, we have for $i=1,2$ :

$$
\int_{\Omega_{\varepsilon}}\left(u_{\varepsilon}^{i}\right)^{2} d x \geq \int_{\Omega_{2 \varepsilon}}\left(u_{i}\right)^{2} d x=1-\int_{C_{2 \varepsilon}}\left(u_{i}\right)^{2} d x \geq 1-\frac{4 \alpha \beta^{2} \varepsilon^{4}}{\cos ^{4} \alpha / 2}
$$

the last inequality coming from (9). In the same way, using the orthogonality of $u_{1}, u_{2}$ in $L^{2}\left(\Omega^{*}\right)$, we get

$$
\begin{gathered}
\int_{\Omega_{\varepsilon}} u_{\varepsilon}^{1} u_{\varepsilon}^{2} d x=\int_{\Omega_{2 \varepsilon}} u_{1} u_{2} d x+\int_{B_{\varepsilon}}\left(\chi_{\varepsilon}\right)^{2} u_{1} u_{2} d x= \\
=-\int_{C_{2 \varepsilon}} u_{1} u_{2} d x+\int_{B_{\varepsilon}}\left(\chi_{\varepsilon}\right)^{2} u_{1} u_{2} d x
\end{gathered}
$$


what yields

$$
\left|\int_{\Omega_{\varepsilon}} u_{\varepsilon}^{1} u_{\varepsilon}^{2} d x\right| \leq 2 \int_{C_{2 \varepsilon}}\left|u_{1} u_{2}\right| d x \leq \frac{8 \alpha \beta^{2} \varepsilon^{4}}{\cos ^{4} \alpha / 2}
$$

We estimate now the integrals with the gradient:

$$
\int_{\Omega_{\varepsilon}}\left|\nabla u_{\varepsilon}^{i}\right|^{2} d x \leq \int_{\Omega^{*}}\left|\nabla u_{i}\right|^{2} d x+\int_{B_{\varepsilon}}\left|\nabla \chi_{\varepsilon}\right|^{2} u_{i}^{2} d x .
$$

As usual, from the construction of a cut-off function, there exists a constant $C$ such that $\left|\nabla \chi_{\varepsilon}\right|^{2} \leq \frac{C}{\varepsilon^{2}}$ and therefore, using one more time (9)

$$
\int_{\Omega_{\varepsilon}}\left|\nabla u_{\varepsilon}^{i}\right|^{2} d x \leq \lambda_{i}+\frac{4 C \alpha \beta^{2} \varepsilon^{2}}{\cos ^{4} \alpha / 2}:=\lambda_{i}+C_{1} \beta^{2} \varepsilon^{2}
$$

In the same way,

$$
\left|\int_{\Omega_{\varepsilon}} \nabla u_{\varepsilon}^{1} \cdot \nabla u_{\varepsilon}^{2} d x\right| \leq \int_{B_{\varepsilon}}\left|\nabla u_{1}\right|\left|\nabla u_{2}\right| d x+\int_{B_{\varepsilon}}\left|\nabla \chi_{\varepsilon}\right|^{2}\left|u_{1} u_{2}\right| d x
$$

what yields (using $\left|\nabla u_{i}\right| \leq \beta$ on $B_{\varepsilon}$ and $\left|B_{\varepsilon}\right| \leq 3 \varepsilon^{2} \tan (\alpha / 2)$ )

$$
\left|\int_{\Omega_{\varepsilon}} \nabla u_{\varepsilon}^{1} \cdot \nabla u_{\varepsilon}^{2} d x\right| \leq \beta^{2} \varepsilon^{2}\left(\frac{4 C \alpha}{\cos ^{4} \alpha / 2}+3 \tan (\alpha / 2)\right):=C_{2} \beta^{2} \varepsilon^{2} .
$$

Taking into account (10), (11), (12) and (13), we get

$$
\lambda_{2}\left(\Omega_{\varepsilon}\right) \leq \max _{\left(a_{1}, a_{2}\right) \in \mathbb{R}^{2}} \frac{\lambda_{1} a_{1}^{2}+\lambda_{2} a_{2}^{2}+\beta^{2} \varepsilon^{2}\left(C_{1}\left(a_{1}^{2}+a_{2}^{2}\right)+2 C_{2}\left|a_{1} a_{2}\right|\right)}{a_{1}^{2}+a_{2}^{2}-\frac{4 \alpha \beta^{2} \varepsilon^{4}}{\cos ^{4} \alpha / 2}\left(a_{1}^{2}+a_{2}^{2}+4\left|a_{1} a_{2}\right|\right)}
$$

what yields (dividing by $a_{1}^{2}+a_{2}^{2}$ and using $2\left|a_{1} a_{2}\right| \leq a_{1}^{2}+a_{2}^{2}$ )

$$
\lambda_{2}\left(\Omega_{\varepsilon}\right) \leq \frac{\lambda_{2}+\beta^{2} \varepsilon^{2}\left(C_{1}+C_{2}\right)}{1-\frac{12 \alpha \beta^{2} \varepsilon^{4}}{\cos ^{4} \alpha / 2}} .
$$

In the same time, $\left|\Omega_{\varepsilon}\right|=\left|\Omega^{*}\right|-\left|C_{2 \varepsilon}\right|=\left|\Omega^{*}\right|-4 \varepsilon^{2} \tan (\alpha / 2)+o\left(\varepsilon^{2}\right)$ and therefore

$$
\left|\Omega_{\varepsilon}\right| \lambda_{2}\left(\Omega_{\varepsilon}\right) \leq\left|\Omega^{*}\right| \lambda_{2}+\varepsilon^{2}\left(\beta^{2}\left(C_{1}+C_{2}\right)\left|\Omega^{*}\right|-4 \lambda_{2} \tan (\alpha / 2)\right)+o\left(\varepsilon^{2}\right) .
$$

Then it is clear that, for $\varepsilon$ small enough, we will have $\left|\Omega_{\varepsilon}\right| \lambda_{2}\left(\Omega_{\varepsilon}\right)<\left|\Omega^{*}\right| \lambda_{2}$ as soon as $\beta^{2}<\frac{4 \lambda_{2} \tan (\alpha / 2)}{\left(C_{1}+C_{2}\right)\left|\Omega^{*}\right|}$ what gives the desired contradiction.

In the sequel, we need to assume that the minimizer $\Omega^{*}$ is a little bit more regular, in order to be able to perform derivative computations w.r.t. the domain. Let us mention that we will prove below that it is at most $C^{2}$.

(H) We assume the minimizer $\Omega^{*}$ to be of class $C^{1,1}$.

By classical regularity results, see [13] or [14], this will imply that the eigenfunction $u$ is $C^{1}$ up to the boundary. 


\subsection{Optimality conditions}

Of course, our main ingredient in the following proofs will be optimality conditions satisfied on the boundary of $\Omega^{*}$. We use again the classical tool of derivative with respect to the domain for the eigenvalues. The difficulty is to take care of the convexity constraint when deforming the original domain $\Omega^{*}$ by a vector field $V$. Indeed, if we perform a small deformation of a strictly convex part of the boundary of $\Omega^{*}$, this part will not remain necessarily convex, but we can use the fact that the difference between the deformed boundary ant its convex hull is so small, that for first order terms, the formulae of derivative still holds (see below for more details). On the contrary, for segments included in the boundary, it is no longer true. Therefore, we need to make a distinction between the strictly convex parts of the boundary and the segments included in the boundary. Let us mention that the first part of this Theorem hold for any dimension while the second part is strictly two-dimensional.

\section{Theorem 7.}

- There exists a positive constant $\alpha$ such that the gradient of the eigenfunction $u$ is constant on every strictly convex part of the boundary of $\Omega^{*}$ :

for every $\gamma$ strictly convex part of $\partial \Omega^{*}, \forall x \in \gamma \quad|\nabla u(x)|=\alpha$.

Moreover $\alpha$ is given by

$$
\alpha^{2}=\frac{\lambda_{2}}{\left|\Omega^{*}\right|} .
$$

- If $\Sigma$ is a segment included in the boundary of $\Omega^{*}$, let $t, t \in[a, b], a$ parametrization of the segment (the boundary is assumed to be oriented in the clockwise sense), then there exists a non negative function $w$ defined on $[a, b]$ with triple roots at $a$ and $b$, such that

$$
|\nabla u(t)|^{2}=\alpha^{2}+w^{\prime \prime}(t)
$$

Proof : We begin by considering $\gamma$ a strictly convex part of the boundary of $\Omega^{*}$. We assume that $\gamma$ is parametrized by a strictly convex function $\varphi$ defined on an interval $I$. We fix a regular function $h$ compactly supported on a sub-interval $J$ and we denote by $\Omega_{\varepsilon}$ the domain (not necessarily convex) whose boundary is (locally) bounded by the graph of the function $\varphi_{\varepsilon}:=$ $\varphi+\varepsilon h$. Let us introduce $\varphi_{\varepsilon}^{* *}$ the convex regularization of $\varphi_{\varepsilon}$ (it is the largest convex function less than or equal to $\varphi_{\varepsilon}$ ) and $\Omega_{\varepsilon}^{*}$ the convex domain whose boundary is (locally) bounded by the graph of the function $\varphi_{\varepsilon}^{* *}$. The key point of the following proof is an estimate given in the Lemma 2 of [23], when the function $\varphi$ is strictly convex :

$$
\left\|\varphi_{\varepsilon}^{* *}-\varphi_{\varepsilon}\right\|_{\infty}=o(\varepsilon) \text { when } \varepsilon \rightarrow 0 .
$$


We now use the quantitative estimate between eigenvalues given in [9] (Lemma 2.1):

$$
\left|\lambda_{2}\left(\Omega_{\varepsilon}^{*}\right)-\lambda_{2}\left(\Omega_{\varepsilon}\right)\right| \leq C\left\|\varphi_{\varepsilon}^{* *}-\varphi_{\varepsilon}\right\|_{\infty}=o(\varepsilon)
$$

(with $C$ a positive constant). Moreover, according to Hadamard formula for simple eigenvalues, see [19], [30]:

$$
\lambda_{2}\left(\Omega_{\varepsilon}\right)=\lambda_{2}(\Omega)-\varepsilon \int_{\partial \Omega^{*}}|\nabla u(\sigma)|^{2} h(\sigma) n_{2}(\sigma) d \sigma+o(\varepsilon)
$$

$\left(n_{2}(\sigma)\right.$ is the second component of the exterior unit normal vector). From (18), (19) we get:

$$
\lambda_{2}\left(\Omega_{\varepsilon}^{*}\right)=\lambda_{2}(\Omega)-\varepsilon \int_{\partial \Omega^{*}}|\nabla u(\sigma)|^{2} h(\sigma) n_{2}(\sigma) d \sigma+o(\varepsilon) .
$$

In the same way,

$$
|| \Omega_{\varepsilon}^{*}|-| \Omega_{\varepsilon}|| \leq \int_{J}\left|\varphi_{\varepsilon}^{* *}-\varphi_{\varepsilon}\right|=o(\varepsilon)
$$

while, by Hadamard formula for areas

$$
\left|\Omega_{\varepsilon}\right|=|\Omega|+\varepsilon \int_{\partial \Omega^{*}} h(\sigma) n_{2}(\sigma) d \sigma+o(\varepsilon) .
$$

Therefore, we also have

$$
\left|\Omega_{\varepsilon}^{*}\right|=|\Omega|+\varepsilon \int_{\partial \Omega^{*}} h(\sigma) n_{2}(\sigma) d \sigma+o(\varepsilon) .
$$

Finally (20), (21) give

$\lambda_{2}\left(\Omega_{\varepsilon}^{*}\right)\left|\Omega_{\varepsilon}^{*}\right|=\lambda_{2}(\Omega)|\Omega|+\varepsilon \int_{\partial \Omega^{*}}\left[\lambda_{2}(\Omega)-|\Omega||\nabla u(\sigma)|^{2}\right] h(\sigma) n_{2}(\sigma) d \sigma+o(\varepsilon)$.

In the previous relation, we can use either $h$ or $-h$, therefore the minimality of $\Omega^{*}$ gives the desired result (14), (15).

Now, let us consider the case of a segment $\Sigma$. The general formula, for the derivative of the function $\Omega \mapsto \lambda_{2}(\Omega)|\Omega|$ at "point" $\Omega^{*}$, according to a perturbation field $V$ is

$$
d\left(\lambda_{2}(\Omega)|\Omega|\right),\left(\Omega^{*}, V\right)=\int_{\partial \Omega^{*}}\left[\lambda_{2}(\Omega)-|\Omega||\nabla u(\sigma)|^{2}\right] h(\sigma) n_{2}(\sigma) d \sigma
$$

In formula (22), the only perturbations $V$ which are allowed are such that the deformed domain $(I d+\tau V)\left(\Omega^{*}\right)$ is still convex (for small $\tau$ ). It is the case if and only if $t \mapsto V \cdot n(t)$ is a concave function on $[a, b]$. Let us denote by $v=V . n$ such a concave function. Replacing in (22) and using (14), (15) yields on the segment $\Sigma$ :

$$
\int_{a}^{b}\left(\lambda_{2}-|\nabla u(\sigma)|^{2} A\left(\Omega^{*}\right)\right) v d t \geq 0 .
$$


Introducing $w_{2}(t)=|\nabla u(t)|^{2}-\alpha^{2}$ it can be also rewritten:

$$
\int_{a}^{b} w_{2}(t) v(t) d t \leq 0
$$

This relation (24) must be true for every (regular) concave function $v$. In particular, in the case $v(t)=1$ and $v(t)=t$, both functions $v$ and $-v$ are concave, therefore

$$
\int_{a}^{b} w_{2}(t) d t=0 \quad \int_{a}^{b} t w_{2}(t) d t=0 .
$$

Now, let us introduce the functions

$$
w_{1}(t)=\int_{a}^{t} w_{2}(s) d s \quad \text { and } \quad w(t)=\int_{a}^{t} w_{1}(s) d s=\int_{a}^{t}(t-s) w_{2}(s) d s .
$$

According to (25), we have $w_{1}(a)=w_{1}(b)=w(a)=w(b)=0$. Integrating twice by parts, it comes

$$
\int_{a}^{b} w_{2}(t) v(t) d t=\int_{a}^{b} w(t) v^{\prime \prime}(t) d t
$$

this last integral must be non positive (according to (24)) for every function $v$ concave, i.e. for every function $v$ such that $v^{\prime \prime} \leq 0$, this yields $w \geq 0$. At last $a$ and $b$ are triple roots of $w$ because $w^{\prime \prime}(a)=w_{2}(a)=0$ by continuity of the gradient $\left(|\nabla u|^{2}-\alpha^{2}\right.$ vanishes identically on the strictly convex parts of $\left.\partial \Omega^{*}\right)$.

A first consequence of the optimality conditions is the fact that the minimizer $\Omega^{*}$ cannot be the "stadium" (convex hull of two identical tangent discs) as it was suggested in the paper of Troesch, [31]. More precisely, we prove:

Theorem 8. The minimizer $\Omega^{*}$ has no arc of circle in its boundary.

Proof : Let us assume that $\partial \Omega^{*}$ contains an arc of circle $\gamma$. We put the origin at the center of the corresponding circle and we introduce the function $w(x, y)=x \frac{\partial u}{\partial y}-y \frac{\partial u}{\partial x}$. Then, we easily verify that

(i) $-\Delta w=\lambda_{2} w$ in $\Omega$

(ii) $w=0$ on $\gamma$

(iii) $\frac{\partial w}{\partial n}=0$ on $\gamma$.

Now we conclude, using Hölmgren uniqueness theorem, that $w$ must vanish in a neighborhood of $\gamma$, so in the whole domain by analyticity. This leads to a contradiction because $u$ would be radially symmetric in $\Omega^{*}$.

Remark 2. The previous result holds in every dimension: the minimizer cannot contain any piece of sphere. The proof is the same using the functions $x_{i} \frac{\partial u}{\partial x_{j}}-x_{j} \frac{\partial u}{\partial x_{i}}$ since $u$ is radially symmetric as soon as all these functions vanish. 


\subsection{Geometry of the minimizer and another regularity result}

Now, if the stadium is not a minimizer for $\lambda_{2}$, it seems that the real minimizer probably looks like a stadium. Some numerical experiments which will appear in [25] confirms that fact. We recall that we have proved in [18] the following properties:

Theorem 9. The minimizer $\Omega^{*}$ has two segments in its boundary and these segments are parallels.

Remark : In the proof of Theorem 9 (see [18]), we only use the optimality conditions. In other words, Theorem 9 is valid for any critical point of the domain functional $\lambda_{2}(\Omega)|\Omega|$.

As a consequence of the above Theorem, we give another regularity result. We recall that we proved in subsection 3.1 that the minimizer $\Omega^{*}$ was at least $C^{1}$. We prove here the counterpart of this result: the minimizer $\Omega^{*}$ is at most $C^{2}$ !

Theorem 10. The minimizer $\Omega^{*}$ cannot be $C^{2, \varepsilon}$, for any $\varepsilon>0$.

Here by $C^{2, \varepsilon}$, we mean classical Hölder regularity: the second derivative of the local maps would be Hölderian of ratio $\varepsilon$.

Proof : Let us assume that $\Omega^{*}$ is $C^{2, \varepsilon}$, for some $\varepsilon>0$. Then, by classical Schauder regularity results for elliptic p.d.e., see e.g. [13] or [14], this will imply that the eigenfunction $u$ is $C^{2}$ up to the boundary. We choose the coordinates axes so that the $x$-axis is parallel to the two segments. Consequently the function $\frac{\partial u}{\partial x}$ vanishes on the two segments. We want to look more precisely at the nodal lines of $\frac{\partial u}{\partial x}$. According to Hopf's Lemma, each boundary point $X$, located on the segments, where $\frac{\partial^{2} u}{\partial x \partial y}$ vanishes, is a starting point of such a nodal line (e.g. if $\frac{\partial u}{\partial x}>0$ in a neighborhood of $X$, since $-\Delta \frac{\partial u}{\partial x}=\lambda_{2} \frac{\partial u}{\partial x}>0$, we have $\left.\frac{\partial}{\partial n}\left(\frac{\partial u}{\partial x}\right)<0\right)$. Now, if $u$ is $C^{2}, \frac{\partial^{2} u}{\partial x \partial y}$ has to vanish at the extremities of the segments by continuity: if $A$ is such an extremity

$$
\frac{\partial^{2} u}{\partial x \partial y}(A)=\frac{\partial^{2} u}{\partial \tau \partial n}(A)=\lim _{B \rightarrow A} \frac{\partial^{2} u}{\partial \tau \partial n}(B)=0
$$

where $B$ is taken on a strictly convex part of $\partial \Omega^{*}$. Moreover, according to the optimality conditions (16), $\frac{\partial^{2} u}{\partial x \partial y}=\frac{\partial}{\partial x}\left(\frac{\partial u}{\partial y}\right)$ has to vanish at least twice inside the segments. Consequently, there are four nodal lines of $\frac{\partial u}{\partial x}$ starting on each segments. Closing these nodal lines, we define at least three nodal domains of $\frac{\partial u}{\partial x}$ strictly contained in $\Omega^{*}$. Now $\frac{\partial u}{\partial x}$ being an eigenfunction associated to $\lambda_{2}$, the Courant-Fischer nodal domain Theorem would lead to the fact that $\lambda_{2}$ is at least the third eigenvalue of a strict subdomain of $\Omega^{*}$, which is a contradiction with the monotonicity of eigenvalues.

Acknowledgements. The authors want to thank the referees who allow to improve significantly the writing of this paper. 


\section{References}

1. G. Alessandrini, Nodal lines of eigenfunctions of the fixed membrane problem in general convex domains, Comment. Math. Helv. 69 (1994), no. 1, 142154.

2. M.S. Ashbaugh, Open problems on eigenvalues of the Laplacian, Analytic and Geometric Inequalities and Their Applications, T. M. Rassias and H. M. Srivastava (editors), vol. 4787, Kluwer 1999.

3. D. Bucur, Regularity of optimal convex shapes, to appear.

4. D. Bucur, G. Buttazzo, Variational Methods in some Shape Optimization Problems, Lecture Notes of courses at Dipartimento di Matematica Università di Pisa and Scuola Normale Superiore di Pisa, Series "Appunti di Corsi della Scuola Normale Superiore", to appear.

5. D. Bucur, G. Buttazzo, I. Figueiredo, On the attainable eigenvalues of the Laplace operator, SIAM J. Math. Anal., 30 (1999), no. 3, 527-536 .

6. D. BuCUR, J.P. Zolesio, $N$-dimensional shape optimization under capacitary constraints, J. of Diff. Eq.,123 n 2 (1995), 504-522.

7. G. Buttazzo, G. Dal Maso, An Existence Result for a Class of Shape Optimization Problems, Arch. Rational Mech. Anal. 122 (1993), 183-195.

8. R. Courant, D. Hilbert, Methods of mathematical physics, Vol. I and II., Interscience Publishers, New York-London 1962.

9. S.J. Cox, M. Ross, Extremal eigenvalue problems for starlike planar domains, J. Differential Equations 120 (1995), 174-197.

10. G. Dal Maso, An introduction to $\Gamma$-convergence, Birkhäuser, Boston, 1993.

11. R. Dautray and J. L. Lions (ed), Analyse mathématique et calcul numérique, Vol. I and II, Masson, Paris, 1984.

12. G. FABER, Beweis, dass unter allen homogenen Membranen von gleicher Fläche und gleicher Spannung die kreisförmige den tiefsten Grundton gibt , Sitz. Ber. Bayer. Akad. Wiss. (1923), 169-172.

13. D. Gilbarg and N. Trudinger, Elliptic partial differential equations of second order, Springer Verlag, New York 1983.

14. P. Grisvard, Elliptic problems in non-smooth domains, Pitman, London, 1985.

15. E.J. Haug, B. Rousselet, Design sensitivity analysis in structural mechanics. II. Eigenvalue variations, J. Structural Mech. 8 (1980), no. 2, 161-186.

16. A. HenRot, Continuity with respect to the domain for the Laplacian: a survey, Control and Cybernetics, vol. 23, n. 3 (1994), p. 427-443.

17. A. Henrot, Minimization problems for eigenvalues of the Laplacian, to appear in Journal of Evolution Equations special issue dedicated to Philippe Bénilan.

18. A. Henrot, E. Oudet, Le stade ne minimise pas $\lambda_{2}$ parmi les ouverts convexes du plan, C. R. Acad. Sci. Paris Sr. I Math, 332 (2001), no. 4, 275-280.

19. A. Henrot, M. Pierre, Optimisation de forme, book to appear.

20. M.V. KELDYš, On the solvability and the stability of the Dirichlet problem, Amer. Math. Soc. Trans., 2-51 (1966), 1-73.

21. E. KRAhn, Über eine von Rayleigh formulierte Minimaleigenschaft des Kreises, Math. Ann., 94 (1924), 97-100.

22. E. Krahn, Über Minimaleigenschaften der Kugel in drei un mehr Dimensionen, Acta Comm. Univ. Dorpat. A9 (1926), 1-44.

23. T. Lachand-Robert, M.A. Peletier, An Example of Non-convex Minimization and an Application to Newton's Problem of the Body of Least Resistance, Ann. Inst. H. Poincaré 18 (2001), 179-198.

24. A. MELAS, On the nodal line of the second eigenfunction of the Laplacian in $\mathbb{R}^{2}$, J. Differential Geom. 35 (1992), no. 1, 255-263. 
25. E. OUdET, Some numerical results about minimization problems involving eigenvalues, to appear.

26. O. Pironneau, Optimal shape design for elliptic systems, Springer Series in Computational Physics, Springer, New York 1984.

27. G. POLYA, On the characteristic frequencies of a symmetric membrane, Math. Z. 63 (1955) 331-337.

28. B. Rousselet, Shape design sensitivity of a membrane J. Optim. Theory Appl. 40 (1983), no. 4, 595-623.

29. J. Simon, Differentiation with respect to the domain in boundary value problems, Num. Funct. Anal. Optimz., 2 (1980), 649-687.

30. J. Sokolowski, J. P. Zolesio, Introduction to shape optimization: shape sensitity analysis, Springer Series in Computational Mathematics, Vol. 10, Springer, Berlin 1992.

31. B.A. Troesch, Elliptical membranes with smallest second eigenvalue, Math. Comp. 27 (1973), 767-772.

32. S.A. Wolf, J.B. Keller, Range of the first two eigenvalues of the Laplacian, Proc. R. Soc. London A, 447 (1994), p. 397-412.

Ecole des Mines and Institut Elie Cartan Nancy B.P. 23954506 Vandoeuvre les Nancy Cedex, France email: henrot@iecn.u-nancy.fr

and

Institut de Recherche MathématiqueAvancée Université Louis Pasteur, 67084 Strasbourg Cedex France email: oudet@irmasrv1.u-strasbg.fr 